\title{
INVESTIGACIONES
}

\section{Trayectorias educativas y laborales de profesionales. Un análisis desde el capital social, cultural y el habitus institucional ${ }^{*}$}

\author{
Educational and labor trajectories in professionals: \\ an analysis from social, cultural capital and institutional habitus

\section{Deisy Chandia Godoy ${ }^{a}$} \\ ${ }^{a}$ Departamento de Ciencias Sociales de la Universidad Católica de la Santísima Concepción. \\ dchandia@ucsc.cl
}

\section{RESUMEN}

En el contexto social y económico actual las trayectorias laborales son menos predecibles y no dependen exclusivamente de una determinada trayectoria educativa. El objetivo de esta investigación fue caracterizar tales trayectorias de los profesionales en relación al capital social y cultural y al habitus institucional. Se realizaron 17 entrevistas en profundidad a profesionales de Concepción, Chile. Se concluyó que las trayectorias tanto educacionales como laborales de los profesionales que entraron a la universidad con un capital social y cultural deficitario tienen en común el discurso del esfuerzo personal, el que se asume como una realidad normalizada, visualizando una postura más individualista sobre la responsabilidad de construir sus propias trayectorias. La institución universitaria se caracteriza por incorporar estrategias de integración asociadas a herramientas académicas, no así de integración a espacios simbólicos y culturales, más bien es un desafío que asume el propio sujeto y que define las trayectorias laborales posteriores.

Palabras claves: profesionales, educación, trabajo, trayectorias.

\begin{abstract}
In the current social and economic context, career paths are less predictable and do not depend exclusively on a specific educational trajectory. The objective of this research was to characterize the trajectories of professionals in relation to their social and cultural capital. 17 in-depth interviews were conducted with professionals from Concepción, Chile. It is concluded that the educational and labor trajectories of professionals who entered the university with a social and cultural capital deficit have in common the discourse of personal effort, which is assumed as a normalized reality, visualizing a more individualistic position on responsibility of building their own trajectories. The university institution is characterized by incorporating integration strategies associated with academic tools, but not integration into symbolic and cultural spaces, rather it is a challenge that the subject assumes and that defines subsequent work trajectories.
\end{abstract}

Key words: professionals, education, labor, trajectories. 


\section{INTRODUCCIÓN}

En el marco de las profundas transformaciones socio-económicas y culturales de las últimas décadas, realizar estudios superiores para luego incorporarse al mercado del trabajo, con el fin de construir una carrera profesional, no puede entenderse como un proceso predecible (Jacinto, 2010; Gil Calvo, 2009). Actualmente se presenta una multiplicidad de transiciones laborales, de diferentes condiciones y niveles de precariedad, desde formas lineales hacia transiciones reversibles y confusas (Pais, 2007). En este contexto, es factible preguntarse por la real implicancia de la educación superior en el tipo de trabajo que realizan los individuos al egresar como profesionales.

Para la teoría de la modernización, la educación es considerada un factor de movilidad positiva, la evidencia, particularmente en Chile, permite realizar algunas consideraciones. Chile ha sido parte de importantes transformaciones en el sistema educativo las últimas tres décadas. Esto se evidencia en la universalidad de la educación básica y secundaria, además del aumento de la tasa de ingreso a la educación superior, de un 7\% en el año 1980 a un 30\% en el año 2009 (Cabrera, 2016). Este proceso de masificación, da lugar a la incorporación de estudiantes de diversos estratos socioeconómicos a estudios superiores. No obstante, este proceso se ha acompañado de la segmentación, expresada en la desigualdad en el acceso a la universidad, además de los problemas de deserción y estratificación de clase referida al ingreso a las universidades (Leyton, Vásquez y Fuenzalida, 2012). Considerando que la segregación en el ámbito de la educación se expresa en todos los niveles de enseñanza, particularmente a nivel universitario (Rodríguez y Padilla, 2017).

Tales argumentos permiten sugerir una menor movilidad social relativa (Cabrera, 2016). Además, la segmentación se expresa en que aquellos estudiantes de sectores socioeconómicos más bajos pueden acceder a carreras con menos retorno económico, lo cual perpetúa las diferencias sociales (Racynski, 2010; Rodríguez y Padilla, 2017).

Las desigualdades en los capitales resultan ser un elemento explicativo de la movilidad social, particularmente en el ámbito de la inserción laboral (Marqués y Gil-Hernández, 2015).

Un título universitario no asegura un buen trabajo, si no va acompañado de un suficiente capital social y cultural. Quienes carecen de este capital son más propensos a acceder a trabajos de menor cualificación o en subempleo (Balint, 2013). Así mismo, se ha sostenido que, si un sujeto cuenta con redes sociales valiosas o contactos sociales amplios, reduce la probabilidad de estar subempleado (Marqués y Gil-Hernández, 2015).

El rol de las instituciones educativas y en particular la universidad es muy relevante en este contexto de segmentación. Ya que se espera que implementen acciones en pro de la equidad e igualdad de oportunidades (Rodríguez y Padilla, 2017).

En atención a los argumentos relatados anteriormente, esta investigación tiene como objetivo caracterizar las trayectorias educacionales y laborales de los profesionales en relación al capital social, cultural y al habitus institucional. Poniendo especial atención en cómo estos sujetos construyen sus trayectorias a partir del capital social y cultural de origen como también del que adquieren en la universidad. 


\section{DESARROLLO}

\subsection{TRAYECTORIA EDUCATIVA Y LABORAL}

Las trayectorias se van configurando y construyendo de manera no lineal a través del tiempo. Permiten entender los procesos de cambio a nivel estructural y que tienen su expresión a nivel micro social mediante las biografías de los sujetos y sus experiencias, enfatizando la permanente interacción entre ambas instancias. Las trayectorias transcurren en el tiempo histórico y en el espacio social en donde se van añadiendo una sucesión de posiciones sociales (Buonotempo, 2000).

Las trayectorias se asocian a recorridos en el ámbito laboral y a actividades profesionales. En los que intervienen aspectos macro, como el mercado laboral y el momento social de egreso, y microsociales, como la familia de origen y la formación educacional (Jiménez, 2009, p. 2). Es decir, son historias en contextos cambiantes, que articulan saberes provenientes de distintos orígenes (Galart y Jacinto, 1995).

Su caracterización requiere que los sujetos estudiados tengan un periodo de permanencia en el campo de trabajo que permita reconstruir las rutas que conforman su desarrollo y actividad profesional, así como analizar los aspectos profesionales, sociales y académicos que han favorecido y/o dificultado dichos recorridos (Jiménez, 2009).

Específicamente las trayectorias educativas y laborales, interrelacionan los recorridos referidos a la formación académica y la experiencia laboral. Jiménez (2009) la define como una instancia en donde se analiza simultáneamente la sucesión de estudios formales e informales que van configurando un desarrollo profesional y el curso que sigue la vida de cada persona en el campo laboral.

\subsection{EL CAPITAL SOCIAL Y CULTURAL, Y HÁBITUS INSTITUCIONAL COMO ENFOQUES TEÓRICOS}

Las trayectorias educativo-laborales han sido analizadas desde diversos puntos de vista, aunque en un comienzo el énfasis estuvo puesto predominantemente en factores socioeconómicos. Sin embargo, a estos indicadores tradicionales deben añadirse el entorno relacional de los sujetos y el trasfondo educativo y cultural que adquieren, y que van situando a estructuras diferenciadas que provienen de la distribución de formas de capital y que dan sentido a la estructura social (Cabrera e Infante, 2016).

El campo es un espacio social de interacción de los participantes o agentes que están organizados en alianzas y con reglas propias. Los sujetos buscan obtener beneficios de esta organización, establecer legitimidad de aquello que han definido como propio del grupo, mejores posiciones, prestigio, o bien desmarcarse de otros grupos.

La posición en el espacio social o campo está sujeta a la cantidad y legitimidad del capital, como también del habitus que vayan adquiriendo los sujetos a lo largo de la vida. En cada espacio social tienen lugar formas específicas de distribución de recursos económicos, sociales, culturales y simbólicos, en donde el sujeto es un participante activo en la construcción de esa estructura social. Por lo tanto, el habitus se conforma a partir de la incorporación que hace el agente de tales estructuras sociales internalizadas (Piñero, 2012). El habitus determina prácticas sociales, estilos de vida, gustos y particularidades propias de un origen social.

Bourdieu (2000) establece la existencia de tres tipos de capitales: económico, cultural y social. Considera el concepto de capital porque se puede acumular a lo largo del 
tiempo y también, en cierta medida, porque permite la transmisión generacional, y, como todo capital, da un poder a su poseedor. Desde el enfoque propuesto por Loury, Granovetter y Bourdieu (citados en Sánchez, 2007) las personas desde la individualidad, se apropian del capital social como recurso que surge en las relaciones sociales.

El capital social es definido como el conjunto de recursos que tiene el sujeto o son potenciales vinculados a la posesión de una red sostenida en el tiempo de relaciones más o menos institucionalizadas de mutuo apoyo o reconocimiento (Bourdieu, 1987).

Esta propuesta enfatiza los beneficios que los sujetos aprovechan de la participación en grupos sociales, con el fin de crear capital social. En este sentido, el capital social constituye un medio para el acceso a otros capitales, como el económico y el cultural (Forni, Siles y Barreiro, 2004).

El capital social a nivel individual se transforma en un elemento facilitador de la obtención de posiciones deseables en la sociedad, especialmente en el ámbito laboral, puesto que el sujeto que posee amplios stocks de este capital tiene la riqueza de las diversas relaciones que ostenta (verticales y horizontales), como también posee, potencialmente, lo que aquellas relaciones le ofertan como oportunidades. En este sentido, el capital social reproduce la desigualdad social, pero también puede contribuir a la integración dentro de grupos específicos.

Según Bourdieu (2011) el capital cultural se presenta de tres formas: la primera se refiere a un estado incorporado, referido a disposiciones durables del organismo; la segunda forma asociada a un estado objetivado, asociado a bienes culturales, libros, cuadros, todo aquello relacionado con el conocimiento; y la tercera forma, expresada en un estado institucionalizado, que se asocia al reconocimiento legitimado, mediante títulos o certificados obtenidos a través de estudios formales.

En relación al capital cultural, Bourdieu (1987) plantea que las instituciones escolares actúan, predominantemente, otorgando títulos y reconocimientos educativos a quienes pertenecen a situaciones culturales, sociales y económicas privilegiadas. Así se legitiman y refuerzan desigualdades sociales de origen, a las que se les asigna un carácter natural de inteligencia. Por lo tanto, el paso por las instancias educativas formales consagra mayoritaria, pero no exclusivamente, a los estudiantes cuyas procedencias familiares, culturales, económicas y de vinculaciones sociales son aventajadas (Sidicaro, 2009).

La escuela contribuye a reproducir el capital cultural, en una estrecha relación entre las estrategias de la familia y de la institución escolar, señalando que es un instrumento de poder a nivel del individuo bajo la forma de un conjunto de cualificaciones intelectuales producidas por el medio familiar y el sistema escolar (Bourdieu, 1987).

En este sentido los conocimientos y valores transmitidos por familias de clase social alta están mucho más asociados a los requerimientos de colegios y universidades, por lo tanto, el aprendizaje resulta ser más natural y facilitador, sustentando su permanencia en la clase dominante (Stillerman, 2016).

El capital cultural, entendido como posesión de bienes culturales, materiales o simbólicos, puede ser determinante en el acceso que tienen los jóvenes a las oportunidades particularmente de tipo educacional (Piñero, 2012). Es decir, los sujetos que no logran un nivel de formación educacional propuesto por la cultura dominante, son excluidos del sistema (Cabrera e Infante, 2016). También es importante considerar que las desigualdades sociales y culturales pueden reducirse en las primeras etapas educativas y en las posteriores transiciones educativas (Kucel, 2011). 
Las trayectorias educativas no tan solo se ven influenciadas por los capitales de origen que tienen los estudiantes, sino también por las disposiciones de las instituciones educativas.

Bal, Davies, David y Reay (2002) y McDonough (1997), proponen la idea del habitus institucional. Referido a las predisposiciones, percepciones y expectativas que conforman las instituciones educativas (Reay et al., 2001). El habitus institucional se origina a partir del vínculo entre agencia y estructura, es decir, la relación entre la clase social de origen y el ámbito socio-institucional.

Esta perspectiva considera que, en estudiantes de la misma posición social, el contexto educacional del que forman parte junto con la clase social de origen, afectan en la proyección futura y el tipo de trayectorias educativas de los jóvenes.

La entidad socio-educativa, por medio de las prácticas cotidianas de los individuos que la componen y su estructura, pueden entregar a los estudiantes más posibilidades o restringirlas (Tarabini, Curran y Fontdevila, 2015). Pudiendo generar, identidad, sentido de pertenencia, compromiso académico y acceso a nuevos capitales sociales y simbólicos (Rodríguez y Padilla, 2017).

Cabe señalar que el habitus institucional es modificable, se transforma y también se adapta a los requerimientos del entorno (Tarabini, Curran y Fontdevila, 2015).

La investigación empírica da cuenta de dos perspectivas centrales frente al habitus institucional: la perspectiva organizacional y la perspectiva culturalista (Leyton, Vásquez y Fuenzalida, 2012; Tarabini, Curran y Fontdevila, 2015).

La perspectiva organizacional se vincula a la cultura organizacional y pone énfasis en las normas y valores de la institución, las concepciones individuales del profesorado mediadas por las prácticas organizativas de la institución escolar y también las expectativas y preparación que tienen los profesores respecto a los estudiantes. En tanto, la perspectiva culturalista tiene que ver con el perfil de selección de estudiantes, las exigencias o demandas educativas, y las vías de apoyo a los estudiantes, que tiene cada institución educativa. Además, considera las características socioeconómicas y culturales de los estudiantes.

En definitiva, no es posible sostener que son solo los estudiantes quienes deciden qué estudiar y dónde estudiar, en base al habitus institucional, sino también las entidades educativas seleccionan a sus estudiantes (Rodríguez y Padilla, 2017).

\section{MÉTODO}

\subsection{METODOLOGÍA}

La presente investigación asume una estrategia metodológica cualitativa, la que permite reconstruir temporalmente la serie sucesos no lineales relatados por los sujetos, profundizando en los sentidos y significados que los actores sociales les dan a sus experiencias (Roberti, 2012), desde sus propios marcos interpretativos (Taylor y Bogdan, 1992). Todo esto desde una estrategia temporal longitudinal y retrospectiva (Hernández, Fernández y Baptista 2014).

\subsection{POBLACIÓN Y MUESTRA}

La población del estudio está compuesta por egresados de una Universidad de la región del Bio-Bio, perteneciente al Consejo de Rectores de Universidades chilenas (CRUCH) 
Estudios Pedagógicos XLVII N 2: 31-51, 2021

TRAYECTORIAS EDUCATIVAS Y LABORALES DE PROFESIONALES. UN ANÁLISIS DESDE EL CAPITAL SOCIAL, CULTURAL Y EL HABITUS INSTITUCIONAL

y de la Red de Universidades Públicas no Estatales pertenecientes al Consejo de Rectores (G9). Su matrícula es de aproximadamente 13.000 estudiantes de pregrado. Concentra principalmente a estudiantes de los tres primeros quintiles socioeconómicos (estrato socioeconómico bajo y medio bajo), según los registros de la Dirección de Admisión y Registro Académico (2019).

El proceso de selección de los participantes se realizó mediante el muestreo no probabilístico (Vieytes, 2004), de caso típico (Izcara, 2007). Se consideraron los siguientes criterios de inclusión: tener más de cinco años de egreso de la universidad y estar trabajando en su profesión o en otra actividad y bajo cualquier modalidad contractual. Además, se tuvo presente el criterio de saturación (Bardin, 2002). De esta manera, la muestra quedó constituida por 17 profesionales (ver tabla 1), cuyas edades oscilaban entre los 30 a los 40 años.

Tabla 1. Muestra

\begin{tabular}{|c|c|c|}
\hline Identificación & Profesión & Especializaciones/postgrados \\
\hline E 1 Mujer & Periodista & - \\
\hline E2 Hombre & Ingeniero Comercial & - \\
\hline E3 Hombre & Ingeniero Comercial & - \\
\hline E4 Mujer & Periodista & Magíster \\
\hline E5 Mujer & Trabajadora Social & Diplomado \\
\hline E6 Mujer & Licenciada en Lenguaje y Periodista & Magíster \\
\hline E7 Hombre & Periodista & Magíster \\
\hline E8 Mujer & Trabajadora Social & Magíster \\
\hline E9 Hombre & Periodista & Magíster \\
\hline E10 Hombre & Ingeniero Civil Industrial & Magíster \\
\hline E11 Mujer & Licenciada en Historia & Magíster-Estudiante Doctorado \\
\hline E12 Mujer & Trabajadora Social & - \\
\hline E13 Hombre & Abogado & Estudiante de Magíster \\
\hline E14 Mujer & Periodista & Doctorado \\
\hline E15 Hombre & Ingeniero Civil Industrial & Doctorado \\
\hline E16 Hombre & Médico & Especialización /Subespecialización \\
\hline E17 Mujer & Nutricionista & - \\
\hline
\end{tabular}

Fuente: Elaboración propia, 2020. 


\subsection{TÉCNICA E INSTRUMENTO}

Se utilizó la técnica de entrevista en profundidad de tipo semiestructurada (Flores, 2009). Se diseñó un guion de temas en relación a los objetivos de la investigación. Cada eje temático consignado ha sido coherente con el fundamento teórico y empírico.

Las categorías generales incorporadas en el guion de entrevista fueron: capital cultural, capital social, trayectorias educacionales, trayectorias laborales, valoraciones y atribuciones de las trayectorias educativas y laborales, e impacto subjetivo de las trayectorias educativas y laborales. El guion fue evaluado por juicio de experto.

Además, se realizó una prueba piloto para revisar la comprensión de las preguntas y el tiempo de aplicación de la entrevista. Paralelamente se procedió a establecer contacto con los participantes para presentarles el objetivo de la investigación, los acuerdos de confidencialidad de la información y los aspectos prácticos de la sesión de trabajo. La duración de las entrevistas osciló en una hora y media.

\subsection{CRITERIOS DE CALIDAD}

Se resguardaron los siguientes criterios de calidad de la información cualitativa: credibilidad, auditabilidad y transferibilidad (Vasilachis, 2006). Además de tener presente las estrategias de verificación de la calidad del proceso de investigación (Morse, 2003). Al finalizar cada entrevista se implementó la validación comunicativa (Flick, 2007).

\subsection{ANÁLISIS DE DATOS}

Se realizó la transcripción de las entrevistas, del formato audio al textual, resguardando íntegramente el discurso de cada participante. Luego, se realizó una lectura y relectura de la información, con la finalidad de comprender el contexto y discurso de los sujetos. El procesamiento de datos textuales se realizó a través del software ATLAS Ti 7. La información fue respaldada en las unidades hermenéuticas de tal programa. Cada entrevista en formato texto fue codificada en base categorías de análisis preestablecidas en relación a los objetivos específicos y argumentos teóricos y empíricos revisados. Con los datos ordenados se dio paso al análisis de dato textual de tipo hermenéutico (Baeza, 2002) centrado en la interpretación en profundidad del texto analizado.

\section{RESULTADOS}

\subsection{CAPITAL CULTURAL Y SOCIAL}

Entre las características socioculturales de las familias de origen de los entrevistados se evidencia que el nivel educativo de los padres y madres se concentra mayoritariamente en la enseñanza básica y media, solo hay dos casos en donde uno de los padres es profesional. Los trabajos que desempeñaban sus progenitores se asociaban a oficios diferenciados por roles tradicionales de género. La mayoría de ellos realizaba trabajos no calificados, sólo en un mínimo de casos realizaba trabajos técnicos y profesionales. En el caso de las madres, trabajaban como: dueñas de casa, asesora de hogar o comerciante, aquellas 
que tenían más años de educación trabajaban como secretaria y técnico en enfermería. Los padres se desempeñaban como: taxista, comerciante, oficial de armada, auxiliar de colegio, eléctrico, entre otros. En general, se caracterizaban por ser familias austeras, cuyos recursos económicos se centraban en la educación y las necesidades básicas. Algunos de los profesionales reconocen situaciones muy precarias a nivel social:

yo me acuerdo que yo estaba en la universidad, y recién pudimos instalar un baño con alcantarillado, entonces durante la básica de verdad que era como un campo... yo tenía vecinos que tenían bueyes, mi abuelita tenía animales... entonces era muy rural (E2).

En el discurso se enfatiza el esfuerzo físico que implicaba el trabajo desempeñado por los padres. Estos recuerdos han sido un estímulo y referente para alcanzar sus objetivos. Tal discurso se vuelve más significativo cuando los entrevistados solo han tenido el cuidado de la madre.

mi mamá se sacrificaba en otras cosas, mi mamá prefería ir a pagarnos el colegio que comprarse zapatos, pagar el colegio en vez de comprarnos ropa a nosotros, yo no tuve auto desde niña, mi mamá tenía el mismo abrigo todo el invierno (E6).

En cuanto a los recursos utilizados para el aprendizaje, reconocen que el uso de medios tecnológicos personales no era masivo. Un grupo de entrevistados comenta que a sus padres les gustaba leer, por lo que en su hogar había libros, revistas o periódicos. Para otros, era imposible tenerlos en su casa.

no teníamos libros, de hecho, yo pude contar con dos profesores que me querían mucho en la enseñanza básica, y me facilitaban libros todas las semanas (E9).

También se evidencia en los relatos el incentivo por la lectura.

Siempre tuvieron en la casa, libros, tenían enciclopedias, compraban Icaritos ${ }^{1}$ cuando yo era chico, que es lo que me fomentaba la lectura, y que yo creo que por eso terminé estudiando derecho (E13).

El contexto familiar da cuenta de la importancia de la participación y pertenencia a grupos sociales activos, ya que forman parte de las actividades cotidianas que definen su entorno social. Es muy frecuente la participación en actividades ligadas a redes institucionales, como la Iglesia Católica y Evangélica, y también a partidos políticos. Igualmente se vinculan a redes con fines lúdico-recreativos, como entidades deportivas y grupos musicales, entre otras.

\subsection{TRAYECTORIA EDUCACIONAL}

La mayoría de los entrevistados provienen de establecimientos municipales, en menor medida, de establecimientos subvencionados y solo dos casos en un colegio particular. En

Icarito fue una revista de apoyo escolar chilena muy popular durante la década de 1980 (N. del E.). 
general, hay un discurso bastante homogéneo en relación al apoyo hacia el estudio recibido por parte de sus padres, el que se manifestaba en la exigencia, el control, el sustento económico y el incentivo a la superación:

en enseñanza básica de lo que me acuerdo, me iba bien, pero no excelente, era un niño tranquilo, que no daba mucho que hacer, era más de cumplir con lo que se me pedía, pero nada más, mis papás trabajaban, eran estrictos conmigo, me apoyaban en los estudios, yo siempre fui bien aplicado, y mi familia me pedía mantener eso nada más...esfuerzo (E16).

yo estudié en una escuela muy pequeñita, partió con dos salas, de hecho, yo fui de los primeros alumnos que estudiaron ahí, era un entorno muy vulnerable, pero a pesar de eso había que surgir y salir adelante (E9).

La experiencia de la enseñanza media la relacionan con el estudio autónomo y mayor preocupación por el rendimiento académico. También la describen como una instancia de crecimiento personal, en donde comenzaron a relacionarse con personas distintas a su entorno más cercano.

En cuanto a la carrera universitaria, se muestra en la mayoría de los participantes que no había una definición previa de esta, más bien visibilizaban áreas de interés. Aunque, finalmente las posibilidades de elección estaban restringidas por los resultados obtenidos en la prueba de selección universitaria, lugar geográfico de la universidad, el costo económico de la carrera y la condición familiar que permitiera financiar los estudios.

al rendir la prueba de aptitud, me fue bien, y ahí descubrí realmente el tema económico en mi casa, yo quedé en kinesiología en Temuco, y ahí mi padre, con mi madre, me contaban cuanto ganaban, mantenían una familia de cinco integrantes, con 210 mil pesos, entonces rápidamente cambié y me matriculé a la carrera que tenía más cerca, periodismo (E9).

En algunos casos, se rendía la Prueba de Selección Universitaria sin mayor expectativa:

yo fui a dar la PSU, y quedé ciento cuarenta y siete de ciento cincuenta, entonces yo dije ¡quedé!, y me aparece fondo solidario, entonces le dije a mis papás, voy a estudiar ahora, no voy hacer el preu, y mi papá me dijo listo, hay que pagar la matrícula, y mi papá vendió una vaca y vinimos para acá y me matriculé y entré (E3).

En relación a la experiencia académica universitaria, en algunos casos se relata que el primer año en la universidad fue complejo porque debían adaptarse a un nuevo sistema de enseñanza aprendizaje. Se evidenciaron las dificultades, ya que no contaban con todas las herramientas para sobrellevar la exigencia propia de la universidad, entre ellas, la deficiencia en inglés, no disponer de hábitos de estudio y vacíos de conocimiento:

yo fui un alumno mediocre, una parte por flojo, y la otra parte porque no tenía las herramientas, no fui formado para la universidad, la mayoría de mis pares fueron formados para la educación técnica... entonces fue tortuoso ese camino a la universidad, pero pude sacar la carrera, en los años que me correspondían, no me atrasé, pero fui un mediocre (E9). 
También se experimenta la percepción de sentirse en desventaja académica y social respecto a otros compañeros:

tuve que congelar un año, porque llegué como a cuarto, y me hicieron inglés, y ahí reprobé inglés, me iba muy mal porque como nunca tuve una base en el inglés, ni en nivel básico, ni en la media, y mis compañeros todos venían de un colegio particular, y muchos se manejaban en el inglés (E1).

Para enfrentar la educación universitaria han sido fundamentales la planificación, el establecimiento de metas y prioridades, y aprovechar cada instancia académica que permitiera aprender, como las ayudantías, participación en investigaciones, y colaboración con los profesores.

me esforcé mucho el primer y segundo semestre, y ahí le encontré el ritmo a esos ramos que todos le tenían miedo a reprobar, traté de dejar de un lado el carrete y la fiesta...iba a mis clases nunca faltaba nada, trataba de participar en lo que podía, aprendi harto, traté de que, si había congresos, o había olimpiadas, yo iba, si había intercambios, lo tomé, y así aprovechar lo que me daba la universidad, y mis compañeros muy geniales la mayoría (E3).

Parte de los entrevistados ha realizado estudios de postgrado, especialmente magíster y dos de ellos doctorado. Otros tienen en proyección realizarlos. Se considera que es importante la especialización, pero una vez que se tenga experiencia laboral, además resultan imprescindible para quienes se dedican a la academia.

siento que nos hemos enloquecido en este mercado del post grado donde, no quiero ser peyorativa, pero sabemos que hoy el mercado de los post grados, se vende, se compra, entonces ya es moda, entonces esta cosa que todo el mundo va por el magíster... sin pensar si tienes las competencias académicas para seguir estudiando y saber a lo que te quieres dedicar (E6).

\subsection{TRAYECTORIA LABORAL}

Para algunos profesionales la trayectoria laboral se inicia paralelo a sus estudios universitarios realizando trabajos temporales en periodo de vacaciones o en algunos momentos específicos del año, para colaborar con los gastos de la universidad.

trabajaba en temporadas recolectando fruta, como dos días atendiendo un minimarket, lo que sí a veces era trabajo de campo, y dentro de la universidad, hacía cosas puntuales, pero no por largos periodos, lo que si en cuarto estuve de empaque como un mes que fue antes de irme a China para tener dinero, y todos los veranos que trabajaba haciendo mi práctica (E3).

Mientras que otro grupo sostiene que sus padres preferían que se dedicaran completamente al estudio para que terminaran pronto su carrera universitaria. 
a pesar de todas las precariedades económicas que nosotros teníamos, mis padres nunca quisieron que yo trabajara... como todos en sus familias salían al mundo laboral, con la enseñanza básica incompleta, con la enseñanza media a lo más, ellos tenían el temor que me gustase la vida laboral, y dejara los estudios de lados, entonces ellos asumieron ese costo y yo lo agradezco (E9).

Si bien la participación en ayudantías o trabajos colaborativos en la universidad recibían una remuneración, no las visualizaban como trabajo propiamente tal, sino como una oportunidad de aprendizaje y de adquirir experiencias, que valoran a medida que han pasado los años.

postulé para ser ayudante, di la prueba pasé y quedé, y ahí me empecé a meter. Los ayudantes de la universidad, como estábamos en el laboratorio, teníamos acceso a internet, entonces aprendí a crear páginas, a buscar información, yo ayudaba a un profe que después guio mi tesis, y él me pasaba una minuta así, y me decía, ya Javier quiero que me traigas archivos de todo esto, y yo se lo pasaba en disquete y le decía ya profe aquí están, y empezaba a trabajar (E7).

El periodo de inserción laboral, una vez que egresan de la universidad, presenta características diversas entre los participantes; para algunos de ellos se trata de un periodo de bastante tensión y espera, en donde no hay redes de contactos que faciliten el ingreso a un empleo:

Apenas egresé, hacía treinta postulaciones de trabajo diaria, me levantaba, entraba a plataformas de postulación, buscaba los cargos, los leía, y empezaba a postular, y bueno haciendo eso, aprendí muchas cosas... después de hacer esto por varios meses, me llamaron a una entrevista y así quedé trabajando en Orizon (E3).

Otros participantes comentan que para ellos fue muy importante la instancia de las prácticas profesionales, ya que les permitió insertarse al trabajo.

El trabajo de la municipalidad, era el mismo centro de práctica que yo hacía, así que les gustó la forma que yo trabajaba, yo era bastante responsable, si de hecho cuando me contrataron, yo todavía no me titulaba de acá, y el alcalde y directora de desarrollo comunitario, me dijeron que el compromiso era que ese año bueno yo en julio o agosto terminaba mi práctica, y en octubre estaba entregando mi tesis, así que me contrataron y no estaba titulada (E8).

En relación a las características que permiten insertarse y permanecer en el trabajo, resultan relevantes la responsabilidad y compromiso con el trabajo, la capacidad de autogestionar el trabajo, y manejar competencias personales para relacionarse con otros.

depende de las habilidades de cada uno, y no tanto del conocimiento que puede adquirir, la forma de empatía, todo parte aquí en la universidad, si tú tienes buena relación con los profesores, apoyas, empiezas a hacer diferentes cosas para que te conozcan, esto te sirve mucho después del egreso, es importante como tú te relacionas 
con las personas, y el compromiso y responsabilidad, yo creo que esa es la base, para ser un buen profesional (E5).

Un discurso bastante generalizado entre los participantes fue sobre lo relevante que se vuelven hoy en día las "redes de contacto" para facilitar los procesos de ingreso al mundo del trabajo.

tú puedes tener muchos méritos, pero es muy complicado, aparte de eso cuando tu egresas te piden experiencia, y tú no tienes experiencia, y como no tienes experiencia y no te la puedes inventar, la única forma de lograr esos puestos, inmediatamente bien pagados, es teniendo alguien que te diga tú vas a estar ahí (E13).

Aunque cabe señalar, que el uso de las redes de contacto también se asocia a una práctica negativa en la sociedad.

soy amigo de las redes de contacto cuando uno ha logrado algo, pero no soy amigo de las redes de contacto para alcanzar algo... para mí el tema de la probidad es importante y si a mí me parece que las cosas no están bien fundadas yo prefiero no darles curso, es decir para conseguir este trabajo vine yo directamente a golpear las puertas (E9).

Para varios entrevistados las redes de contacto tienen lugar en el espacio universitario, sobre todo cuando desde la familia de origen no hay vínculos con grupos que tienen otros capitales.

Son importantes las redes que uno genera, primero porque yo cuando estaba en la universidad me creé redes, y me ayudó a poder acceder a tener mi propia empresa de ingeniería, donde yo era mi propio jefe, en el que iba a las empresas a prestar servicios, después que trabajé en Huachipato, e hice un buen trabajo, entonces ellos me recomendaron a otras empresas... (E15).

Para algunos participantes, la inserción al campo laboral se hace más compleja al no poder ejercer su profesión, buscando otras alternativas,

después de terminar quinto, en la tesis estuve como un año, también me costó terminar, porque también me puse a trabajar, pero no en periodismo, si no en algo de administración, contabilidad, como había estudiado eso en el liceo, como que siempre estuve trabajando en eso, en la parte de números, así que no estudié ningún post grado, ni nada, después, con el tiempo como no encontraba trabajo, decidí quedarme en el área contable (E1).

También relatan algunos entrevistados, que los primeros trabajos a los que accedieron se caracterizaban por darse en condiciones precarias, es decir sueldos muy bajos, con gran inestabilidad, horarios poco definidos, con desgaste físico y realizando tareas muy diversas.

primero empecé a trabajar por amor al arte, ayudaba en unos proyectos de investigación, a una profesora que me hizo clases, no me pagaban, era como para 
aprender, pero después no podía vivir del aire, así que empecé a buscar trabajo, y mis primeros trabajos fueron reemplazos en unos colegios (E11).

Otros participantes desde un principio realizaron actividades laborales de manera independiente, ya que contaban con apoyo y redes.

el primer trabajo que tuve fue de ejercicio libre de la profesión, puse una oficina con harto esfuerzo, el esfuerzo de mis papás, y ese fue el primer trabajo que yo tuve, y lo mantuve hasta el año pasado (E13).

antes de salir de la universidad yo tenía mi propia empresa, y hacía trabajos particulares, como por ejemplo a Huachipato, a Mininco, a ERCO, a todas esas empresas yo comencé a prestarles servicios (E15).

También se muestra la experiencia de aquellos que han entrado al mercado laboral sin mayores dificultades, principalmente por las características de la profesión y del mercado laboral.

en el hospital X me llamaron enseguida, porque gracias a Dios mi profesión tiene una alta demanda entonces no es que cueste mucho conseguir trabajo, es más bien que llegan las ofertas, porque hay mucha gente enferma, y a veces los centros de salud no dan abasto, por tanto, siempre se hacen falta médicos en todos lados (E16).

En relación a la posibilidad de cambiarse de trabajo, la motivación es para optar a un mejor ingreso y alcanzar mayor estabilidad económica, principalmente cuando se ha trabajado sin un contrato que garantice continuidad de trabajo en el tiempo.

bueno yo trabajaba acá y en otras partes a honorarios, entonces uno quiere una estabilidad económica, uno va creciendo, quiere tener familia, y comprarte una casa, y te piden tu renta, te piden años de antigüedad y en todas era a honorario (E10).

Una parte de ellos han trabajado sus primeros años bajo contrato de honorario ${ }^{2}$. Mientras que otros profesionales han cambiado su situación contractual derivando a contratos indefinidos, y al mismo tiempo les ha permitido consolidarse como profesionales.

En otros casos se han limitado a quedar con contrato indefinido, pero solo de media jornada y el resto del tiempo al cuidado de los hijos, principalmente en el caso de la mujer profesional.

ahora trabajo con 22 horas, y no trabajo en ningún otro lugar ahora, antes estuve haciendo clases y algunas asesorías en el ámbito de recursos humanos, pero hasta que quedé embarazada, decidí quedarme con estas 22 horas, así que trabajo toda la mañana y en la tarde estoy con mis hijos (E8).

El contrato a honorarios no considera una relación de subordinación y dependencia entre empleador y trabajador. No se rigen por el Código del Trabajo de manera que no les asiste ninguno de los derechos que tal normativa establece. Se rige por las leyes civiles. (Dirección del trabajo, Gobierno de Chile: www.dt.gob.cl). 
Hay un grupo que no ha logrado estabilidad. Estos han asumido, en cierta manera, tal condición adaptando sus vidas a los ingresos variables y a la inestabilidad del empleo. Comentan que es complejo plantearse la vida en esta condición principalmente por la ansiedad e incertidumbre frente al futuro. Si bien se proyectan en conseguir un trabajo más estable, ven con cierta desesperanza lograrlo.

hay que ser súper honesto, porque hay muchos profesores en historia, y los colegios, cada vez requieren dos profes de historia, cuatro como máximo, las horas laborales, o jornadas completas son pocas, siempre son 22 o 32 horas, entonces se ve complejo el campo laboral, en cuanto a la universidad es un poquito más complejo el ingresar, por la competencia que se da entre los mismos docentes, y por la preparación que tienen los mismos docentes (E11).

Para otros, su proyección está en consolidar un trabajo independiente: “ mi proyección a futuro es poder tener mi trabajo independiente, entonces, poner algún centro de mediación, o un staff de asesoramiento" (E12).

\subsection{VALORACIONES DEL PROCESO EDUCATIVO}

Realizar estudios universitarios ha significado una "oportunidad de acceso" a un espacio tangible y también a un espacio simbólico. Lo tangible se asocia al acceso económico que ha permitido mayor estabilidad económica y proyección en la planificación de la vida. Aunque para quienes no han logrado obtener un trabajo estable, las proyecciones familiares y adquisición de una vivienda no logran concretarse. En general, la educación ha significado optar a un mejor ingreso.

acceder a estudios universitarios, es esencial... para mí el trabajo es el medio, para poder hacer lo que a mí me gusta, la parte que viene después que yo salgo de aquí, o sea si yo tuviese que limpiar baños, para poder hacer lo que a mí me gusta, acceder a la universidad tuvo una importancia fundamental para poder ganarme las lucas (E4).

Respecto al acceso a espacios simbólicos, los participantes reconocen que estudiar en la universidad les ha permitido acceder a espacios de relaciones sociales distintas a la red de origen.

eso hizo romper el círculo de la pobreza...me generó muchísimas más oportunidades de las que tuvo mi madre y mis tíos, para que decir mis abuelos, entonces yo vengo de la típica familia de abuelo obrero, madres y tíos técnicos...me permitió conocer gente muy distinta a la que yo acostumbraba, personas de distintas áreas, etc. (E5).

Como también el acceso a un espacio cultural del cual no eran parte. Se refieren a un sentimiento de valoración en la sociedad, es decir, ser importante por el rol que se cumple como profesional y el reconocimiento.

la posibilidad de tener un mejor pasar que mis viejos, que afortunadamente por la pega de mi papá no era malo lo que se ganaba en ese caso, pero exponencialmente es aumentar ingresos, es aumentar cultura, el poder salir, el poder viajar, el poder 
disfrutar, tener 30 años y tener cosas que a mis papás les tuvo que haber costado 20 años en tener, hoy día yo las puedo tener, pero más que eso es la oportunidad de interactuar con más gente que tú puedes ayudar (E13).

Existe un reconocimiento del discurso que enfatiza las condiciones económicas deficientes en las que ellos crecieron. La vivencia actual, en donde se tiene una mejor situación económica, siempre es contrastada con las experiencias del pasado: "el antes" en donde existían los recursos mínimos para vivir y "el ahora" en donde se han superado barreras sociales y económicas.

De manera menos recurrente, tiene lugar un discurso asociado al significado de acceder a la universidad como una tradición y estatus de familia. Discurso propio de una segunda generación universitaria (continuista): "acceder a la universidad, era como lo mínimo, ya que en mi familia varios tienen estudios superiores" (E8).

En cuanto a la valoración del proceso educativo, se fortalece la idea del "esfuerzo", principalmente asociada a la historia familiar. También se valora la relación con los profesores, destacando en ellos la confianza, el apoyo y el incentivo para continuar preparándose en la vida profesional.

también tuve muy buenos profes, yo creo que tuve facilitadores que fueron esenciales, como para formarme académicamente con algunos hábitos que fueron bien fundamentales, y aquí en la universidad tuve uno o dos profesores, que fueron fundamentales en eso, en ir abriendo un poco los ojos, uno llega sin saber mucho y ahí hay profes que a uno la apadrinan, armándole camino (E4).

Entre los obstáculos del proceso educativo, los entrevistados destacan las deficiencias en la comprensión de textos, manejo de lenguaje técnico y aprendizaje de otros idiomas, principalmente inglés. Lidiar con tales obstáculos fue un esfuerzo, pero en el presente se valoran como un aprendizaje.

\subsection{VALORACIONES DE LA TRAYECTORIA LABORAL}

Es posible reconocer tres aspectos que les han permitido configurar sus trayectorias laborales: Un primer discurso asociado a las características personales como la capacidad de superación y perseverancia, como una disposición interna del sujeto para lograr metas, a pesar de las limitaciones las que se adquieren en la familia de origen y en las experiencias personales.

yo se lo debo a mi constancia, a mi propio esfuerzo, a nunca bajar los brazos, porque sí, siempre van a existir dificultades, y cosas que nos puedan hacer decaer, pero eso no ha influido en mí de manera negativa, de hecho, me ha ayudado para poder ser mejor, para ser mejor profesional, pero también para ser mejor persona, más integral, y eso también es importante, mi determinación (E15).

El segundo discurso se refiere a la habilidad del sujeto para atender a las oportunidades del entorno laboral, esto se asocia a la capacidad de adaptación a trabajos diversos y a la formación como profesional. 
yo lo valoro mucho el trabajo, o sea sin duda, yo creo que todo es por algo, yo estuve mucho tiempo sin pega, y llegué a un lugar que no me gustó, con gente que no me gustaba, pero lo que rescato de eso, es que ahora valoro todo, y creo que cualquier trabajo es valorable, que es bueno partir de abajo (E4).

El tercer discurso dice relación con la valoración de la red de contacto profesional y nuevos vínculos sociales que les provee el espacio universitario. Este se sitúa como la primera plataforma para incursionar en el nuevo contexto laboral.

todos me conocían, sabiendo que yo era buena alumna me recomendaban para trabajos... los mismos profes me llamaban y me decían oye sabes que, hay un trabajo en Panguipulli, y yo iba (E17).

Las trayectorias laborales se van construyendo a través del esfuerzo y la persistencia. En este recorrido deben tomar una serie de decisiones que les han permitido planificar la vida. Hay quienes piensan que es más fácil emprender el camino profesional y sus trayectorias laborales sin responsabilidad familiar, ya que implica mayor dedicación en tiempo que según ellos no podrían ceder. Postergando la maternidad o paternidad y la conformación de familia.

yo no quiero tener hijos, hasta poder tener un contrato jornada completa, porque hago clases hasta las diez de la noche, entonces yo digo pucha con un hijo, no puedo hacer clases hasta las diez de la noche, pero tampoco las lucas me van a dar para no hacer clases hasta las diez de la noche y tener un hijo, entonces afecta (E4).

me encantaría ser mamá, no puedo tener un hijo porque mi vida laboral, económica, sobre todo, no lo permite, y porque también aún sigue ese estigma que la mujer con hijos, puede ser un cacho, si se abre un concurso, entonces no me sirve, y eso es lo que a mí me frena (E11).

Otros profesionales han ido construyendo sus trayectorias laborales a la par de la consolidación de una familia. El periodo de estudios de postgrado lo asumen con hijos o al menos con una pareja estable. Manifiestan que sin el apoyo y contención familiar no habrían alcanzado los distintos desafíos. Una preocupación central por parte de estos profesionales es la dedicación de tiempo a su familia. También señalan el desgaste emocional que han significado lidiar con el desarrollo académico y la consolidación familiar.

con el doctorado fui dejando de lado a la familia, yo estoy casado, y tengo una hija de siete años... estaba tan mentalizado en terminar mi doctorado, que le comencé a dar más importancia a aquello, por otra parte, seguía trabajando en la universidad, entonces me quitaba todo el tiempo, pero mi familia, mi esposa, me apoyaba en todo lo que estaba haciendo, así que teniendo su apoyo es más fácil para mí también (E15).

En relación al significado que le otorgan al trabajo es posible situarlos desde dos categorías: la primera asocia al trabajo como un medio para el logro de metas. Aquí el trabajo no tiene un fin en sí mismo. El trabajo es una instancia basal que permite proyección 
y organización de la vida: "el trabajo es funcional, es la vía para poder lograr lo que a mí me gusta" (E4).

La segunda categoría considera el trabajo como un espacio relevante para el desarrollo personal, que provee el crecimiento y aprendizaje. El trabajo es parte de la identidad del sujeto. Es un ámbito que permite considerar a la persona de manera integral: "yo creo que el trabajo es importante en la vida del ser humano, porque uno proyecta lo que es" (E6).

\section{DISCUSIÓN Y CONCLUSIONES}

En lo concreto, los profesionales entrevistados provienen de sectores socioeconómicos medios y medios bajos, cuyos padres proveían de manera austera a sus necesidades básicas. Solo uno de los entrevistados era estudiante de segunda generación o continuista, los demás forman parte de una primera generación universitaria. Son los padres quienes transmiten formas de asumir la vida y son constantes referentes para los hijos, traspasando estrategias de afrontamiento ante situaciones adversas.

El esfuerzo y la perseverancia, como características heredadas por la familia, se mantiene en el relato de los profesionales, como una forma de hacer frente a las desigualdades del sistema. Estas se hicieron tangibles en el espacio educativo sobre todo cuando ingresaron a la educación universitaria. Manifestándose en las posibilidades restringidas ante la decisión de estudiar una determinada carrera universitaria y la universidad a la que podrían acceder, como también en la carencia de competencias académicas que les permitieran asumir una educación universitaria en igualdad de condiciones respecto a estudiantes provenientes de otros grupos socioeconómicos.

El discurso "centrado en el esfuerzo personal" es asumido por los profesionales como parte de una realidad normalizada. Es decir, la idea de sacrificio que hicieron sus padres, asociada a la superación de la pobreza, desgaste físico y postergación personal, continúa en el discurso e imaginario de los hijos. Si bien, no se ven enfrentados a tales dificultades, deben superar otras barreras de desigualdad (redes de contacto, oportunidades). Cabe destacar que, frente a esta realidad asumida, no está presente un discurso crítico respecto a la sociedad de la que son parte. Se visualiza así una postura más individualista sobre la responsabilidad de construir sus propias vidas.

Las decisiones que los sujetos van tomando en la configuración de sus trayectorias educacionales se realizan, mayoritariamente, en base a las posibilidades situacionales y económicas. En este contexto, las decisiones vocacionales no son una prioridad, o bien las perspectivas de futuro las van adecuando a sus posibilidades, que tienden a ser restringidas. Incluso la proyección en la formación profesional se enmarca en una disposición a pensar su futuro dentro de los límites culturales y sociales en que crecieron (Castillo y Cabezas, 2010; Rodríguez y Padilla, 2017).

Para quienes son la primera generación de estudiantes universitarios, si bien la universidad les otorga un capital institucionalizado mediante certificado de alguna profesión, esto no es lo más relevante. La universidad es valorada por una característica simbólica referida a la "posibilidad de acceso" a un entorno social y económico del cual estaban excluidos.

Aunque los entrevistados logran reducir las desigualdades sociales y culturales, a través de las transiciones educativas (Kucel, 2011), esto ha implicado un esfuerzo personal, 
de constante superación frente a la situación de desventaja de la que se sienten parte permanentemente. En este sentido reconocen que la institución educativa, si bien considera algunas instancias de integración con el fin de consolidar algunos aspectos académicos básicos, tales no son suficientes. Esto porque persisten a la base códigos culturales y simbólicos que no se superan solo con los aspectos académicos.

Así, la institución educativa mantiene su habitus institucional (Leyton, Vásquez y Fuenzalida, 2012) orientado a incorporar aprendizajes profesionales que tienen como meta la obtención de un certificado.

No obstante, los entrevistados destacan una instancia que permite mayor adaptación y confianza con la cultura universitaria, refiriéndose al vínculo generado con los profesores. Estos son las figuras más cercanas en su formación profesional y con quienes fomentan la interacción social desarrollando habilidades sociales y cognitivas. Son ellos quienes les orientan en sus potencialidades y también les vinculan con un nuevo espacio social.

Para los entrevistados es muy relevante la enseñanza de habilidades no cognitivas, ya que el certificado de un título universitario no hace la diferencia a la hora de consolidarse en el ámbito laboral. Desde sus experiencias, la universidad no logra nivelar a sus estudiantes en tales habilidades, lo que resulta ser una de las dificultades más importantes al incorporarse al campo laboral.

La evidencia analizada permite concluir que las trayectorias laborales que han conformado los profesionales, son el resultado de acontecimientos e interacciones entre aquellos capitales heredados y las oportunidades del contexto. Como también de las significaciones en torno a su recorrido educacional y laboral que permiten modelar aquellas diferencias originadas por las desigualdades de base. Principalmente la económica que restringe los espacios de decisión del sujeto. Quienes han logrado una trayectoria tanto educacional como laboral más exitosa han sido quienes superaron las barreras socioculturales (integración a otros grupos sociales), detectaron oportunidades y asumieron riesgos en pro de un beneficio mayor.

A continuación, se describen tres tipos de trayectorias definidas a partir de las experiencias de los participantes.

1. La trayectoria inestable, centrada en profesionales que no tienen un trabajo estable y que trabajan principalmente a honorarios. En este grupo no se observa una planificación del desarrollo profesional. Las decisiones en el ámbito laboral están más asociadas a un factor económico. La planificación de la vida en varios aspectos es más compleja, al no tener un trabajo con mayor proyección y seguridad. Si bien en su trayectoria laboral han realizado trabajos en distintas áreas, y más flexible en tiempo y tareas, es más bien visto como una desventaja. Ya que les afecta en la consolidación de metas personales.

2. La trayectoria caracterizada por el desarrollo profesional, vinculada a profesionales que han logrado insertarse en el mercado laboral ejerciendo su profesión. Algunos dependientes de un empleador y otros que han consolidado un trabajo independiente como auto empleados. Hay profesionales con postgrados, si bien ha significado un periodo de inversión de tiempo y recursos, pero lo valoran positivamente. El trabajo es valorado para acceder a recursos que permiten una buena calidad de vida, pero también adquiere sentido en la identidad como profesionales. En general, han construido sus trayectorias laborales a la par de la consolidación 
familiar. No obstante, el poco tiempo dedicado a la familia es uno de sus mayores cuestionamientos. También es posible identificar a profesionales que ven en su trabajo actual una instancia de tránsito hacia una etapa de mayor consolidación, como es el caso de aquellos profesionales que están desarrollando un trabajo no vinculado a su profesión o bien más inestable, pero que les permite costear estudios de postgrado. Se observa una planificación de su desarrollo profesional, ya que asumen como una responsabilidad personal la incorporación a un trabajo más estable y mejor remunerado.

3. Y, por último, las trayectorias laborales en donde no se hace ejercicio de la profesión en el campo laboral. Es posible advertir que la identificación con la profesión no está presente en el quehacer diario, solo se valora de manera simbólica y genérica el "ser profesional". Son profesionales que sienten un desencanto con la profesión e intentan desmarcarse de esta, ya que no les ha proporcionado la gratificación esperada en el ámbito económico o bien no lograron insertarse en el campo de trabajo de la profesión durante los primeros años.

De las experiencias relatadas por los profesionales es posible establecer tres ámbitos relevantes en la construcción de las trayectorias laborales y educacionales:

- La desigualdad social cotidiana a la que se enfrentaron en el ámbito educacional y laboral, producto de sus capitales social, cultural y económico; excepcionalmente uno de los entrevistados, no se evidencia tal discurso, ya que forma parte de una segunda generación universitaria.

- Enfatizan la importancia que tienen las entidades educativas universitarias en disminuir la brecha de desigualdad percibida por los estudiantes. Específicamente en el ámbito de las habilidades no cognitivas, que pueden facilitar la impronta de cómo enfrentarse, tanto al espacio académico de la universidad, como también al mundo laboral.

- La importancia de "pertenencia a la universidad", ya que varios profesionales provenientes de sectores sociales más bajos, declaran que en los primeros años de universidad se auto percibían excluidos, al no conocer códigos sociales y culturales propios de la universidad.

Las limitaciones de la presente investigación se relacionan con la heterogeneidad de la muestra de estudio en relación a las profesiones de cada individuo, lo que impide delimitar trayectorias más específicas. Y en relación a la muestra, fue focalizada en profesionales provenientes de solo una universidad. En futuras investigaciones se sugiere estudiar las trayectorias laborales de profesionales mediante una estrategia metodológica cuantitativa y longitudinal, lo que aportaría perfiles de trayectorias más precisos. También sería interesante abordar tipologías de trayectorias particulares que surgieron en este estudio, como sujetos que estén desempeñándose en condición de subempleo. 
Estudios Pedagógicos XLVII N 2: 31-51, 2021

TRAYECTORIAS EDUCATIVAS Y LABORALES DE PROFESIONALES. UN ANÁLISIS DESDE EL CAPITAL SOCIAL, CULTURAL Y EL HABITUS INSTITUCIONAL

\section{REFERENCIAS BIBLIOGRÁFICAS}

Baeza, M. (2002) De las metodologías cualitativas en investigación científico social. Diseño y uso de instrumentos en la producción de sentido. Concepción: Editorial de la Universidad de Concepción.

Bal, S., Davies, J., David, M. \& Reay. D. (2002) Classification and judgement': social class and the "cognitive structures" of choice of higher education. British Journal of Sociology, 23(1), 51-72.

Balint, B. (2013). The role of social capital in avoiding over-education. Revista Romana de Sociologie, 1(2), 101-126.

Bardin, L. (2002) Análisis de contenido. Madrid: Editorial Akal.

Bourdieu, P. (1987). Los tres estados del capital cultural. Revista sociológica, 2(5). . (2000). Las formas del capital. Capital económico, capital cultural y capital social: Poder, derecho y clases sociales. Bilbao: Editorial Desclée de Brouwer. . (2011). Las estrategias de la reproducción social. Argentina: Editorial Siglo XXI.

Bourdieu, P. \& Passeron, J. (2003). Los Herederos. Los estudiantes y la cultura. Ed. Buenos Aires: Siglo XXI.

Buonotempo, M. (2000) Inserción laboral de graduados universitarios. Un estudio desde las trayectorias laborales. Universidad Nacional del Nordeste-Facultad de Relaciones Laborales, Comunicación Social y turismo, Corrientes (Argentina).

Cabrera, M. \& Infante C. (2016). Capital Social Estructural y Educación, un Estudio desde la Perspectiva de Pierre Bourdieu: Estudio de Caso Universidad ECCI. Revista Academia y Virtualidad 9(1), 105-122. http://dx.doi.org/10.18359/ravi.1704

Castillo, J. \& Cabezas, G. (2010). Caracterización de jóvenes primera generaciónen educación superior. Nuevas trayectorias hacia la equidad educativa. Revista Calidad en la educación, (32), pp. 44-76.

Flick, U. (2007). Introducción a la investigación cualitativa. Madrid: segunda edición. Ediciones Morata.

Flores, R. (2009). Observando Observadores: una introducción a las técnicas cualitativas de investigación social. Santiago: Ediciones UC. Forni, P., Siles, M. \& Barreiro, L. (2004). ¿Qué es el capital social cómo analizarlo en contextos de exclusión social y pobreza? JSRI Research Report \#35, The Julian Samora Research Institute, Michigan State University, East Lansing, Michigan.

Galart, M. \& Jacinto, C. (1995). Competencias laborales: tema clave en la articulación educacióntrabajo. Curso subregional de formación de gerentes de educación técnico-profesional, Cinterfor, Montevideo.

Gil Calvo, E. (2009). Trayectorias y transiciones: ¿Qué rumbos? Revista de Estudios de Juventud, (87), 15-30.

Hernández, R., Fernández, C. \& Baptista. P. (2014). Metodología de la investigación. México: sexta edición. McGraw-Hill.

Izcara, P. (2007). Introducción al muestreo. Editorial Miguél Ángel Porrúa. México.

Jacinto, C. (2010). Elementos para un marco analítico de los dispositivos de inserción laboral de jóvenes y su incidencia en las trayectorias. En: La construcción social de las trayectorias laborales de jóvenes: políticas instituciones, dispositivos y subjetividades, por Claudia Jacinto (comp.), 15-49. Buenos Aires: Teseo/ IDES.

Jiménez, M. (2009). Tendencias y hallazgos en los estudios de trayectoria: una opción metodológica para clasificar el desarrollo laboral. Revista Electrónica de Investigación Educativa, 11(1), 1-21.

Kucel, A. (2011). The sociology of educational is match. Polish Sociological Review, 173(1), 21-34.

Leyton, D., Vásquez, A. y Fuenzalida, V. (2012). La experiencia de estudiantes de contextos vulnerables en diferentes instituciones de educación superior universitaria (IESU): resultados de investigación. Calidad en la Educación, (37), 61-97. 
Marqués, I. \& Gil-Hernández, C. (2015). Origen social y sobreeducación en los universitarios españoles: ¿es meritocrático el acceso a la clase de servicio? Revista Española de Investigaciones Sociológicas, 150(1), 89-112. http://dx.doi.org/10.5477/cis/reis.150.89

McDonough (1997). Choosing colleges: how social class and schools structure opportunity. New York: State University of New York.

Morse, J. (2003) (Coord). Asuntos críticos en los métodos de investigación cualitativa. España: Editores Universidad de Antioquía.

Olmedo, A. (2007). Reescribiendo las teorías de la reproducción social: influencia de la clase social en las trayectorias educativa y laboral del alumnado granadino de secundaria y bachillerato. Revista de educación, 343, 477-501.

Pais, J. (2007). Chollos, chapuzas y changas. Jóvenes, trabajo precario y futuro. Barcelona: Antrhopos.

Piñero, S. (2012). Jóvenes universitarios: desigualdades socioculturales y diversidad de representaciones en torno a la profesión. Revista Liminar Estudios Sociales y Humanísticos $X(1), 33-45$.

Raczynski, D. (2010). Hacia una estrategia de validación de la educación pública municipal: Imaginarios, valoraciones y demandas de las familias. Santiago de Chile: Ministerio de Educación, Fondo de Investigación y Desarrollo en Educación.

Reay, D., David, M. y Ball, S. (2001). Making a difference? Institutional habitus and higher education choice. Sociological Research Online, 5(4).

Roberti, E. (2012). El enfoque biográfico en el análisis social: claves para un estudio de los aspectos teóricos-metodológicos de las trayectorias laborales. Revista Colombiana de Sociología 25(1), 127-149.

Rodríguez, C. \& Padilla, G. (2017). Elección profesional y sesgo de selección: evaluación de los sistemas de admisión universitaria en Chile en un contexto de agenda pro-inclusión. Revista da Avaliação da Educação Superior, 22(03), 852-870.

Sánchez, R. (2007). La teoría de los campos de Bourdieu, como esquema teórico de análisis del proceso de graduación en posgrado. Revista Electrónica de Investigación Educativa, 9(1).

Stillerman, J. (2016). Educar a niñas y niños de clase media en Santiago: capital cultural y segregación socio territorial en la formación de mercados locales de educación. Revista Latinoamericana de Estudios Urbano Regionales 42(126), 169-186.

Sidicaro, R. (2009). Reflexiones sobre los sistemas escolares y las desigualdades sociales latinoamericanas en una época de crisis de sentido. En: Diversidad cultural, desigualdad social y estrategias de políticas educativas. Tenti Fanfani (comp.) IIPE UNESCO.

Taylor, S. J. y Bodgan, R. (1992). Introducción a los Métodos Cualitativos de investigación. Buenos Aires: Paidós.

Tarabini, A., Curran, M. \& Fontdevila, C. (2015). El habitus institucional: una herramienta

Teórica y metodológica para el estudio de la cultura escolar. Revista Tempora, 18, 37-58.

Vasilachis, I. (2006) Estrategias de investigación cualitativa. Gedisa: Barcelona.

Vieytes, R. (2004). Metodología de la investigación en organizaciones, mercado y sociedad. Buenos Aires: Editorial de las Ciencias. 
